\title{
Exploratory Methodology for Identification of Urban Bottlenecks Using GPS Data
}

\author{
Andres L. Jimenez ${ }^{1}$ and Alvaro Rodriguez-Valencia ${ }^{2}$ \\ 1. Independent consultant \\ 2. Department of Civil and Environmental Engineering, Universidad de los Andes, Bogotá 111711, Colombia
}

\begin{abstract}
Bottlenecks have been widely studied for uninterrupted flow. The study of bottlenecks in urban traffic networks implies a challenge, since delays and queuing are implicit in interrupted flows. The objective of this paper is to discuss bottlenecks in urban traffic networks and present a methodology to identify them, based on GPS (Global Position System) data from taxis in Bogotá, Colombia. In networks, where stops are frequent, the principle we adopted deals with finding recurrent low-speed sections, beyond expected delays. Urban bottlenecks occur in those road network segments that perform poorly in terms of speed, compared with upstream and downstream conditions, producing recurrent and larger than normal delays. The GPS devices reported, via GPRS (General Packet Radio Service), information every $10 \mathrm{~s}$. Results of this exploratory project are promising. The method allowed the identification of six urban bottlenecks out of seven randomly selected low-speed sections. The most valuable application of this methodology is the prioritization of resources investment in traffic infrastructure improvements. As a low cost option, this exploratory method might be especially attractive in identifying critical points in traffic networks in developing cities, without expensive traffic-monitoring systems.
\end{abstract}

Key words: Urban bottleneck, bottleneck, road network, GPS, congestion, investment prioritization, traffic performance.

\section{Introduction}

Urban bottlenecks have been less studied than bottlenecks in free-flow segregated traffic, e.g., motorways. However, UBs (urban bottlenecks) are of increasing importance as congestion worsens in urban areas and measures to use more efficiently the existing infrastructure have become mandatory options prior increase in capacity. Many of the common physical elements causing bottleneck, such as lane drops or merge areas, are also present in urban networks. However, much less research efforts have been devoted to study this phenomenon in cities.

Congestion is a symptom of economic growth [1]. Traffic congestion in urban areas is often present in cities with vibrant economic development, which results in more employment, more activities, and more interactions. That make people want to move more and faster. Rapid growth in personal income (GDP (gross

Corresponding author: Alvaro Rodriguez Valencia, assistant professor, research field: transport systems. domestic product) per capita) causes the well-recognized growth in automobile ownership [2-4], as well as an increase in mobility rates [4]. Along with present urban population growth, which is much more rapid in developing countries, these trends result in a considerably increase in urban traffic.

Congestion occurs when the speed on the road decreases and delays arise. Congestion adds costs to the movement of people and freight mainly in terms of lost time. Two broad groups of definitions of congestion are frequently found in the literature: the first is related with the macro-level demand for road use. Congestion appears when demand exceeds capacity [5]. The second group of definitions is related with the difference between the roadway system performance that users expect and how the system actually performs. Thus, congestions management also tracks for travel reliability, in addition to average travel speeds. Similarly, the European Report for Congestion also identifies two principal broad categories of causal 
factors for congestion: macro-level factors that relate to overall demand for road use and micro-level factors (e.g., those that relate to traffic "on the road") [1].

Bottlenecks are associated with congestion. Bottlenecks have been widely studied for uninterrupted flow. When congestion occurs in a specific location (not along the entire corridor), a bottleneck may exist. An active freeway bottleneck is a point in the road network characterized by the presence of queued traffic immediately upstream and unrestricted (unqueued or freely-flowing) traffic downstream $[6,7]$.

While the literature on freeway bottlenecks is robust, there are limited references on this phenomenon in urban roads. The main problem might be the difficulty in defining what can be considered as a UB, since at-grade intersections frequently produce temporary queues with unrestricted flowing conditions up- and down-stream. The issue is how to distinguish between a regular low-speed section and a UB. Not considering every single intersection as a UB, but identifying those critical points in the network is the goal. Therefore, we define a UB as a section in the urban network, with recurrently low-speeds (preceded and followed by good traffic conditions), where delays exceed the reasonable waiting times. Similar to freeway bottlenecks, lane drop, potholes, and merging areas can produce these situations. A bad performance in a single section of a network may affect several other links, causing an inefficient use of the infrastructure. By improving these sections, the specific bottleneck disappears, and the network performance will increase. This means that resources can be efficiently invested.

In this paper, we concentrate on the micro-level factors, specifically on bottlenecks in urban networks. This paper contributes in exploring bottlenecks in urban areas and introduces the routine we applied to identify the bottlenecks using GPS (Global Position System) data. While traditionally bottleneck locations are usually identified through direct observation, aerial photographs, video surveillance data, or dynamic surveillance on freeways equipped with detection technology [8], this identification approach presents several advantages. Among the most remarkable advantages are the suitability for extensive networks, the applicability in cities with no traffic counting and monitoring systems, and the low costs. Among the specific applications of the identification of bottlenecks in urban areas are the efficient investment of anti-congestion resources and efforts.

The methodology was successfully applied in a case study in Bogotá, Colombia for identifying UBs. This methodology is suitable for extensive urban networks (big cities) with poor traffic counting and monitoring systems and restricted financial resources.

\section{Literature Review}

Bottlenecks are considered an important research topic in the field of traffic engineering since bottlenecks can be a major source of delays in road traffic [8]. Bottlenecks are part of many motorway systems [9] and can occur in different situations: upgrades, lane drops, merge areas, waving sections [10], tunnels, underpasses, narrow lanes, lack of shoulders, curves and poorly operated traffic signals [8]. The capacity of a road depends on many factors, some intrinsic of the facility itself (e.g., number of lanes) and other temporal (e.g., rain). This means that the capacity of the road might not be constant along the whole network and throughout the day.

Along with the demand, the road capacity determines the delays [11]. A bottleneck is usually activated when the increase in demand causes the saturation of the bottleneck, thus leading to the formation of queues. Several authors have found that the capacity in bottlenecks drops once a queue has formed upstream, compared with those measured prior to queuing [6, 11-13]. If queues could be delayed or avoided in the presence of a bottleneck, extra capacity would be available [13].

Bottlenecks can occur at fixed locations [14], in variable locations [15], or can be produced temporarily (e.g., by incidents, in which capacity is reduced by the 
blocking of lanes) $[8,11,16]$. So, they can be classified as static or dynamic. Recurring bottlenecks are those, which activate daily at the same hour of time almost every day [17]. Moving bottlenecks happen when a slow-moving vehicle (e.g., a truck or any oversized vehicle) disrupts the continuous flow of the general traffic [18]. This can reduce the capacity of the network's roads and intersections, thus affecting negatively travel times [18].

\section{Methodology and Data Source}

Bogotá has a very serious traffic congestion problem. According to Acevedo et al. [19], the total amount of automobiles in Bogotá will rise from 700 thousand in 2008 to 3.6 million in 2040. The annual growth rate of the road infrastructure in Bogotá has been only $0.49 \%$ for the last 5 years. However, the current infrastructure seems to be sufficient to provide a decent service, based on the relationship between the vehicle fleet and lane-kilometer. This indicator was still low compared with most North American cities (and similar to London) [19]. The poor traffic management and deficiencies in existing infrastructure has led to an inefficient use of the road infrastructure in Bogotá. For instance, Bogotá has more than 1,000 traffic lights, most of them still operating in fixed-times (very few actuated signals).

Direct observation to traffic conditions in Bogotá permitted the identification of situations similar to bottlenecks. By means of processing and analyzing a vast GPS database, we were able to develop an urban bottleneck identification routine. This routine was simultaneously developed when analyzing the data. However, for the sake of clarity, we present first the methodology in an abstract way, followed by its application using the Bogota GPS data base.

A vast GPS database of GPS data were analyzed. The data came from a pilot project for traffic congestion monitoring in real-time (SITYMUR ("Sistema de información de trafico y movilidad urbana" in Spanish)) in Bogotá of Andes University
(Universidad de los Andes). The GPS data were acquired from four devices installed in different taxis during two months (June and July, 2010). The GPS devices reported, via GPRS (General Packet Radio Service), information every $10 \mathrm{~s}$. The resulting GPS dataset includes records for each individual vehicle, containing the taxi ID, the longitude, the latitude, the date, and the time. Taxis travel an average of $250 \mathrm{~km}$ per day, per shift [20]. In addition, it was also proven that there is no significant speed difference between empty and occupied taxis in Bogotá [21]. The data set has data for all days in the week (except Sundays), especially from 6:00 a.m. to 8:00 p.m.

\section{Description of the Bottleneck Identification Approach}

With the purpose of identifying UBs, the principle we applied deals with finding recurrent low-speed sections in the network, preceded and followed by higher speed zones. These sections are segments of the road network that perform poorly (in terms of traffic speed) compared with upstream and downstream conditions, producing recurrent delays. Unlike free-flow traffic infrastructures, traffic in urban roads is intrinsically characterized with stops. Aware of that, one of the further discussions on this paper is which delay is "larger than normal" that constitutes an urban bottleneck. Thus, we use the space-mean speed as the metric to identify UBs.

This approach was developed from an existing GPS database acquired for traffic analysis in Bogotá. The data came from GPS devices installed in three taxis tracking position regularly in time (every $10 \mathrm{~s}$ ). GPS produces a succession of location points subsequently distributed in time that produces a polyline that represents the trace of the car in the network. Spatially, the closer the points are to each other, the lower the speed of the vehicle. Our goal deals with identifying these low-speed sections and recognizing which are caused by extraordinary sources (understanding ordinary causes these that produce expected stops and 
their associated delays).

In this section, we present the procedure in a general/abstract manner, and in the next section, we will present an applied example (case study) in Bogotá. The steps of the methodology are: (1) database revision and cleanup; (2) speeds calculation; (3) identification of low speed sections; (4) identification of UBs; and (5) on-site visit to determine the source of the bottleneck. We started up from an existing database, therefore, the methodology does not include any insight of how to acquire the data efficiently (number of cars and hours of data acquisition, etc.).

\subsection{Step 1: Data Revision}

GPS devices-tracking positions in short time intervals for days-produce huge amount of measurements (latitude $X$, longitude $Y$, height $Z$, and time $T)$. An $i$ th measurement is described:

$$
M_{i}=\left(X_{i}, Y_{i}, Z_{i}, T_{i}\right)
$$

Identifying missing or wrong measurements is important to have continuous use of all data in time. Missing data are inconvenient since the methodology is based on mean speeds, calculated using measurements $M_{i}$ before or after. Imputations are straightforward, since time is varying in a regular manner (every $10 \mathrm{~s}$ ) and the location is likely to be on the network between the points $10 \mathrm{~s}$ after and before. By depicting the data, it is also possible to identify those, which clearly divert from a reasonable polyline. While GPS devices have increased in accuracy, still there are sources of errors, especially in urban areas, where high-rise buildings and other constructions can distort the right coordinates of the measurement $M_{i}$. This step mostly deals with the treatment of blanks, checks of extreme values and outliers, imputation of missing or wrong information, and correcting of measurements. Detailed information about the database cleanup process can be found in Bravo et al. [22, 23].

\subsection{Step 2: Speed Calculations}

Low-speed sections are identified by means of speeds, using time intervals and distances. Time intervals are constant (10 s), but we can use any bigger time interval multiple of $10(20 \mathrm{~s}, 30 \mathrm{~s}$, etc.). Here $k$ works a as a counter (integer number) to describe the number of intervals back and forth (each interval is of the minimum timespan, $10 \mathrm{~s}$ in this case). For example, $k=1$ or $k=-1$ refers to preceding and following points, respectively. If $k>0$, points are ahead in time and in $k<0$, point are before in time. Time differences for a measurement $n$ is calculated:

$$
t_{i, k}=T_{i+k}-T_{i}
$$

where:

$t$ is the time difference between the $i$ th and the $(i+k)$ th measurements (lower case represent differences);

$T$ is time for a measurement $i$ (capitalized represent absolute time values);

$k$ is the number of $10 \mathrm{~s}$ intervals. If $k=2$, it means the point $20 \mathrm{~s}$ after the measurement $i$;

$i$ is the number of the measurement.

The distance between consecutive points (length of each segment in the polyline) is calculated by processing position information, namely longitude $\left(X_{i}\right)$, latitude $\left(Y_{i}\right)$ of two consecutive points (for flat terrain we assumed $Z_{i}$ constant for all measurements $M_{i}$ ). The calculation requires the transformation from the WGS84 coordinate system (the reference coordinate system used by the GPS) into plain coordinates in Bogotá. After those transformations, distance $d_{i, 1}$ associated to the given measurement $M_{i}$ and the following point $M_{i+1}$ is function of its coordinates and calculated by applying the regular Pythagorean theorem (for convention, the distance associated to the measurement $i$ is calculated with respect to the next consecutive point in time $i+1)$.

$$
d_{i, 1}=f\left(\left(x_{i}, y_{i}\right) ;\left(x_{i+1}, y_{i+1}\right)\right)
$$

The total distance between non-consecutive points $T_{i}$ and $T_{i+k}$ is the sum of the lengths of the $(i+k-1)$ segments between consecutive measurements.

$$
d_{i, k}=\sum_{k=1}^{k} d_{(i+k-1), 1}
$$


Space-mean speeds can be calculated using time differences $t_{i, k}$ and polyline length $d_{i, k}$. This implies that many speeds can be calculated, depending on the selected reference point $i$ and the time interval $k$ of the second measurement $M_{i+1}$.

$$
\begin{gathered}
\bar{V}_{i, k}=\frac{d_{i, k}}{t_{i, k}} \\
\bar{V}_{i, k}=\sum_{k=1}^{k} d_{(i+k-1), 1} /\left(T_{i+k}-T_{i}\right)
\end{gathered}
$$

For instance, we obtain the closest result to the actual instantaneous velocity by using the shortest time interval, and this comes from the differences between two consecutive measurements $i$ and $i+k$ (where $k=1$ ).

\subsection{Step 3: Identification of Low Speed Sections}

Low-speed sections are defined as segments in the network that have a substantially lower average speed than the upstream and downstream traffic. A low speed section does not necessarily represent a bottleneck, but an active bottleneck always results in a low-speed section. Low speed sections can be identified graphically or analytically. Graphically, a slow-speed section can be easily identified by the succession of several consecutive points very close to each other, proceeded and followed by more spaced points. However, since we have a big sample of points, it would be very time-consuming.

Analytically, low speed zones can be identified by means of the distance between two consecutive points, namely the speed (since $t_{i, 1}$ is always $10 \mathrm{~s}$ ). Similarly when mean speeds are calculated using non-consecutive points.

Different values of $k$ for the speed calculation produce different speed profiles ( $v$ - $t$ graphs). Notice that for lower $k$ values the speed profile resembles the instantaneous speed of the vehicle. On the contrary, for larger $k$ values, small fluctuations are averaged and the speed-time curve is smoother. To make out point clearer, we suggest the reader to check the case study in Fig. 1. Fig.1 shows the mean speed calculated from the shortest interval $k=1$ (or $10 \mathrm{~s}$ ) to $k=30(5 \mathrm{~min}$ ) and shows that the speed fluctuations are less abrupt (or smoother) for greater values of $k$.

\subsection{Step 4: Filtering Special Low Speed Sections}

We classified low-speed sections in two groups: operational or non-operational. For instance, quieting for a traffic light is related with the operation of the vehicles, while stopping for lunch is not. The first task deals with identifying and discarding these non-operational low-speed sections. After that, the second task is distinguishing between ordinary low-speed sections or low speed sections due to ordinary operation in the urban network (e.g., a traffic light) and extra-ordinary low speed sections that produce UBs.

The identification of non-operational stops was a long but straightforward task. Very rarely a vehicle stays steady for a prolonged time on a road. Even less frequent if this occurs in a secondary road. Therefore, we identified short distance differences for consecutive points for prolonged period of time, then we looked the location on the map, and then we analyzed the situation. Maintenance stops, such as fueling, eating, or resting show this pattern.

The distinction between ordinary operational low-speed sections and bottlenecks is rather much difficult to identify. Initially, we intuitively decided to analyze $v$ - $t$ graphs using different values of $k$. Our analysis based on realizing the differences on $v-t$ profiles for different $k$. For example, for greater $k$ values, a short operational stop (30 $\mathrm{s}$ in a regular traffic light) would have small effect on the space-mean speed, i.e., this stop will reduce the mean speed, but will not generate a dramatic bump in the graph. Similarly, a substantial reduction of speed in an extraordinary queue or several consecutive stops will represent a substantially drop in the mean-speed speed calculated with great values of $k$. Conversely, small values of $k$ have a more detailed $v-t$ profile, which requires a more interpretative and intuitive skills to identify a bottleneck. On the other hand, generalized network 


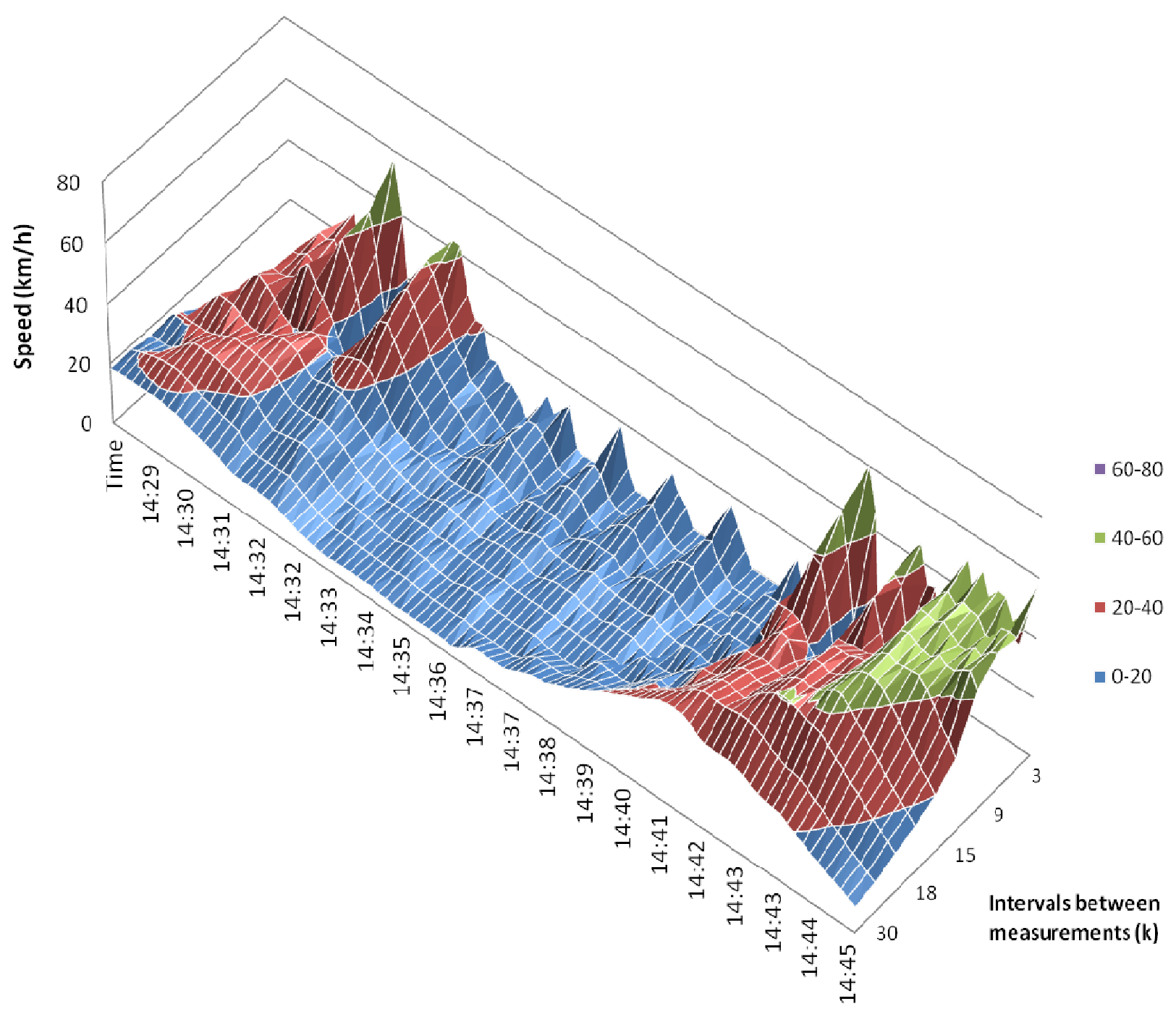

Fig. 1 Space-mean speed profiles for different time intervals.

congestion can also be easily identified. We ended up choosing $k=30$ as a result of doing the whole process for different values of $k$.

\subsection{Step 5: Identification of Urban Bottlenecks}

So far, the methodology permits detecting low-speed sections and discard those that are caused by non-operational stops. In the previous step, the method sorted only the low speed zones that might be potential $\mathrm{UB}$, but there is no certainty whether or not they are bottlenecks. Ideally, more information of the same link from different days will increase the probability of certainty in the identification of pure UB. Repetition of low-speed zones in the same location, at different times and different days would serve as a useful indicator to identify bottlenecks. But since our data were obtained from another project, and we had no power to design a tailored data acquisition procedure to obtain recurrent measurements, we had to include a visual identification process (field inspection). The process we applied for the identification of UB is:

(1) Plotting in GIS (Geographic Information System) these points of the low speed section plus the points of the up and downstream section, in order to observe the bad traffic conditions (low speed sections surrounded by normal flow regimes upstream and downstream). We recommend plotting 50 measurements upstream and 50 downstream of the low-speed point location. 
Approximately, 50 data correspond to $8 \mathrm{~min}$;

(2) Making a field inspection to corroborate the existence of this type of traffic phenomena and to identify the cause of the UB.

\section{Application of the Methodology}

Using the previously explained methodology, it was possible to locate 124 low-speed sections for Bogotá using the previously described taxi GPS dataset. Nineteen of these sections were identified as bottlenecks. In the following sub-sections, we present the stepwise procedure for Bogotá.

\subsection{Step 1}

Although the vast majority of data is complete (few missing data), there are some cases where missing or wrong information is contained. For Step One, the most frequent errors we found were small diversions in time and missing data.

The regularity of the time intervals is very high. Sometimes, especially in centric areas with high buildings, some few measurements can have one second of delay (not cumulative). Very seldom measurements are missing. In any case, since we are working with the actual time differences, this fact does not affect the final output. Therefore, we decided not to correct the time values. Missing data are filled out using the location of the midpoint between the previous and following value and matching it to the network.

Consecutive points $(k=1)$ permits a reasonably clear identification of some events. In order to identify non-operational low speed sections, first we programmed the spreadsheet identify periods where the taxi stayed in standstill state for $5 \mathrm{~min}$ or more. It means identifying 60 or more consecutive points where the distance was $d_{i, k}<10 \mathrm{~m}$ (a stay GPS still may produce non-coincident coordinates, rather a small variation up to some meters might appear).

During the plotting of the polylines, we noticed that it would be possible that the polyline does not coincide with the actual road network (e.g., the polyline cuts off in corners). For the SITYMUR project, we proved that the polyline formed by joining together several segments of consecutive points $\left(d_{i}\right)$ leads to a fairly close approximation of the real trace (less $2 \%$ shorter than the real distance). Therefore, no map matching procedure was applied, especially considering the exploratory scope of this project.

\subsection{Step 2}

We calculated different speeds using different values of $k$. Notice that for greater values of $k$, more points ahead are required, making $(N-k-1)$ points usable. Fig. 1 is an example of the different speed profiles.

\subsection{Steps 3 and 4}

What characteristics should have the traffic flow in order to be a potential UB? Initially, we thought that a ratio of the speed of the upstream (or downstream) and the speed of the low speed section would identify the bottleneck. However, before we could even try, we noticed that a prolonged mean speed around $4 \mathrm{~km} / \mathrm{h}$ would effectively identify UBs. A $\pm 20 \%$ bandwidth was arbitrarily defined, showing good results. We use an algorithm in order to identify sequential points with $\bar{v}_{i, k}$ between $3.2 \mathrm{~km} / \mathrm{h}$ and $4.8 \mathrm{~km} / \mathrm{h}$. Consequently, if this condition is accomplish, a low-speed section is defined. Fig. 2 shows one example of one the algorithm for checking the inequalities. We have chosen, from trial and error, what would be the mean speed threshold that would be useful to identify UB.

\subsection{Step 5}

As mentioned, using the previously explained methodology, it was possible to locate 124 low-speed sections for Bogotá and 19 of these sections were identified as bottlenecks. Due to resource constraints, and being an exploratory work, only seven sections were selected for verification (field observation). Table 1 summarizes the cases, with the precise location and the source of the bottleneck. It is important to 


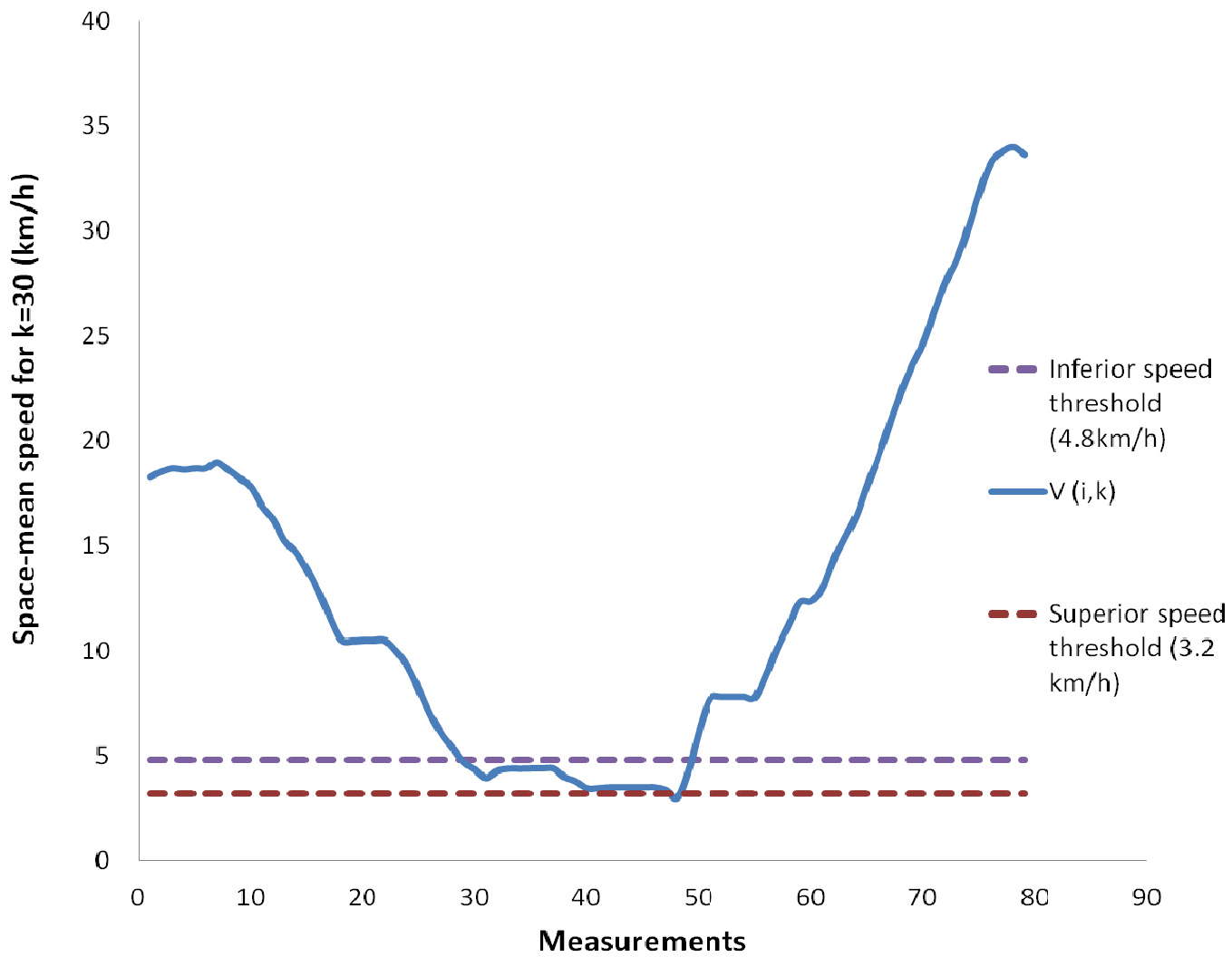

Fig. 2 UB identification (each measurement $k=1$ is equivalent to $t=10 \mathrm{~s}$ ).

Table 1 Sample of potential urban bottlenecks is Bogotá.

\begin{tabular}{llll}
\hline Case & Address & Cause & Success? \\
\hline 1 & Av. Calle 3 x Av. Carrera 68 & Traffic light & Yes \\
2 & Av. La Esperanza x Av. Rojas & Road design (geometry) & Yes \\
3 & Av. Las Américas x Av. Boyacá & Weaving & Yes \\
4 & Av. Boyacá x calle 60A sur & Unknown & No \\
5 & Av. Calle 13 x carrera 47 & Merging traffic & Yes \\
6 & Av. NQS x Av. Carrera 68 & Weaving & Yes \\
7 & Av. NQS x Av. Boyacá & Weaving & Yes \\
\hline
\end{tabular}

remark that the GPS data were acquired in 2010. Therefore, it could be possible that some of the identified UB do not exist in 2011 when we developed and applied the methodology (because Bogotá has undergone major construction works in the network).

Although the essence of the UB identification procedure is to find sections of low speeds, this is not enough for automatic detection in urban networks. This step aims to dismiss the low speed sections that may produce slow traffic as a consequence of non-operational causes (maintenance, dinner, resting etc.). These types of stops are characterized by complete standstill conditions for a reasonable amount of time. Notice that in UB, it is expected at least some movement in the direction of traffic.

Having the plain coordinates, we imported the data to GIS and displayed them on the road network. Fig. 3 shows the results of one section that was classified as a potential UB. As marked on the graph, it is easy from the consecutive ID to identify the flow direction, and to identify the starting point of the queue. Notice as well that there exist all three different traffic states necessary to consider this point as a UB. 


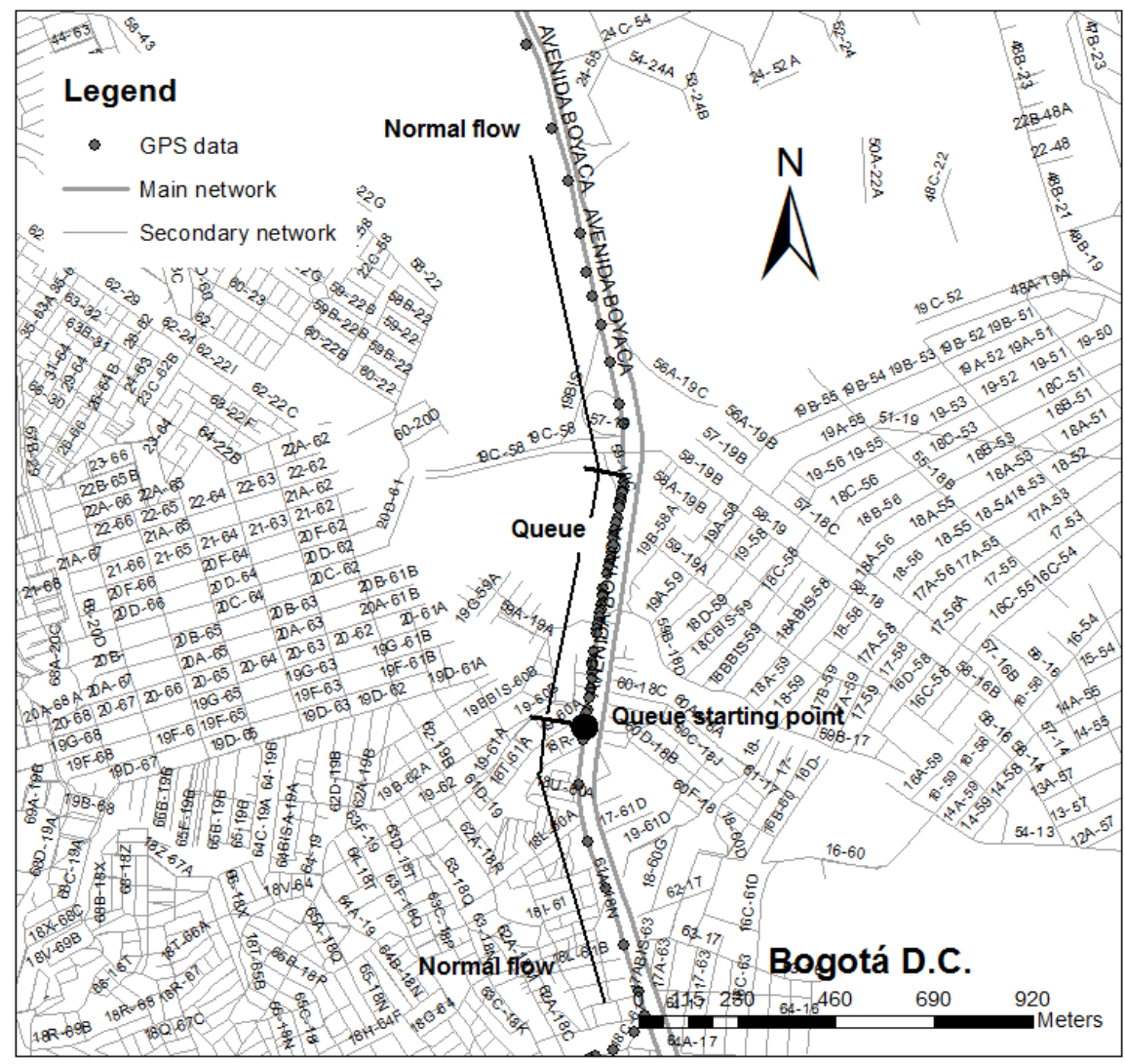

Fig. 3 Space-mean speed profiles for different time intervals.

\section{Conclusions}

The presented routine appears as an economic, feasible, and effective way to identify urban bottlenecks (as defined in the introduction). This empirical routine arises as a valuable tool to identify critic points in urban networks for improving traffic conditions. This is especially valuable in big cities in developing cities like Bogotá, which have limited resources for monitoring traffic systems. The presented method shows several strengths, especially in terms of costs and flexibility. The on-the-way data acquisition devices (loops and sensors) provide localized information (fixed). These devices are robust for highways, where traffic is concentrated in one section. In extensive urban networks, it would be necessary to install hundreds or thousands of these type of devices, to make it possible to uncover UBs.

For the specific case of Bogotá, our routine was able to predict urban bottlenecks in 6 out of the 7 studied cases. The reasons for the long queues and extraordinary delays were a traffic light (1 case), bad road geometry (1 case), weaving zones (3 cases), merging traffic (1 case). Except the traffic light, all cases are to be approached

GPS data, conversely, permits the coverage of the entire network. Since GPS devices send the information in real time, engineers also did not depend on third parties to get the information. Obviously, the on-the-way devices acquire other type of information that GPS cannot get.

Fig. 3 summarizes the potential output of our methodology. There are many things that could be analyzed from these data. Queue lengths and delays can be easily calculated and thus assessments about the negative effects might also be defined, e.g., increase in emissions, fuel consumption, loss of productivity, etc.

In general, bottlenecks are identified and studied in 
order to find ways to overcome their negative effect in traffic. Initially, traffic management (signs and signals) and light interventions (pavement painting) were desired to improve the situation, but sometimes "heavy" infrastructure would be the only way to solve the situation. Looking at the identified bottlenecks in this research (Case 1), there are many possibilities, such as installing actuated (responsive) traffic lights, widening the approaches or constructing an underpass. In Cases 3, 5 and 7, including one or several lanes, or channelizing some flows would avoid the worst traffic conditions. The possible outputs of the vehicle tracks allow us to obtain valuable information to feed cost-benefit analyses for decision-making.

The results are promising. The use of GPS data in the urban bottleneck localization seems viable. For us, one of the most valuable application is prioritizing the investments of scarce resources in traffic infrastructure in an efficient way. This method permits us to compare among dozens of points that would require intervention.

We want to point out that because data were acquired for another project, and we were not able to acquire information under a planned route scheme of the tracking vehicle, we had very few recurrent data (cases of the same section at the same time from different days). Recurrent measurements in same streets at the same hour in different days reduce uncertainty on the distinction of UB, since it is very unlikely to have an external random event (broken car) every day at the same hour in the same place. The field inspection that we applied for this research is an alternative solution to this constrain. In fact, operating taxis use a lot the secondary network, and avoid congestion.

The limited resources and the fact that we started from existing data (acquired for a different purpose) limited our capacity to gather tailored information (e.g., data on the same place at the same hour of the day for different days of the week). In addition, some of the parameters were defined by a trial and iterative process that led to the successful identification of bottlenecks. Although it would be interesting to scientifically define or justify the founded thresholds and parameters, these are beyond the scope of the objective of this research. More work on this would be required in order to develop and refine a reliable, robust methodology.

\section{Acknowledgments}

The authors appreciate the support of the Universidad de los Andes, the Grupo de Estudios en Sostenibilidad Urbana y Regional (SUR) for the GPS data provided for this study. The contribution of Prof. G. Bravo and Ing. L. Bautista was invaluable to make data manageable. In addition, we want to thank the instructor P. Perez for all the support with coordinates transformations and his constant work to make the GPS devices work properly. Last, but not least, we want to express our gratitude to Prof. J.P. Bocarejo, director of the research group SUR.

\section{References}

[1] Joint OECD/ECMT Transport Research Centre. 2007. Organisation for Economic Cooperation and Development. A report of European Conference of Ministers of Transport, Paris, France.

[2] Christidis, P., Hidalgo, I., and Soria, A. 2003. Dynamics of the Introduction of New Passenger Car Technologies: The IPTS Transport Technologies Model. Report of European Commission.

[3] Cameron, T. J., Lyons, and Kenworthy, J. R. 2004. "Trends in Vehicle Kilometers of Travel in World Cities, 1960-1990: Underlying Drivers and Policy Responses." Transport Policy 11: 287-98.

[4] Gómez-Gélvez, J. A., and Obando, C. 2013. "Modeling Car Ownership in Urban Areas of Developing Countries Case Study of Bogotá, Colombia." Transportation Research Record: Journal of the Transportation Research Board 2394 (2013): 111-8.

[5] Schafer, A., and Victor, D. G. 2000. "The Future Mobility of the World Population." Transportation Research Part A 34 (2000) 171-205.

[6] Kutz, M. 2004. Handbook of Transportation Engineering. New York : McGraw-Hill.

[7] Zhang, L., and Levinson, D. 2004. "Some Properties of Flows at Freway Bottlenecks." Transportation Research Record: Journal of the Transportation Research Board 1883 (2004): 122-31.

[8] Daganzo, C. 1997. Fundamentals of Transportation and Traffic Operations. Oxford: Pergamon. 
[9] Federal Highway Administration. 2009. Recurring Traffic Bottlenecks: A Primer. U.S. Department of Transportation.

[10] Wieczorek, J., Li, H., Fernández-Moctezuma, R. J., and Bertini, R. L. 2009. "Integrating an Automated Bottleneck Detection Tool into an Online Freeway Data Archive." Presented at 88th Annual Meeting of the Transportation Research Board, Washington, D.C., USA.

[11] Banks, J. H. 1990. "Flow Processes at a Freeway Bottleneck." Journal of the Transportation Research Board 1287 (1990): 20-8.

[12] Knoop, V. L., Hoogendoorn, S. P., and Van Zuyl, H. J. 2008. "Capacity Reduction at Incidents: Empirical Data Collected from a Helicopter." Journal of Transportation Research Record 2071 (2008): 19-25.

[13] Cassidy, M. J., and Bertini, R. L. 1999. "Observations at a Freeway Bottleneck." A research paper, University of California at Berkeley.

[14] Hall, F. L., and Agyemang-Duah, K. 1991. "Freeway Capacity Drop and the Definition of Capacity." Transportation Research Record: Journal of the Transportation Research Board 1320: 91-8.

[15] Cassidy, M., and Bertini, R. 1999. "Some Traffic Features at Freeway Bottlenecks." Transportation Research Part B: Methodological 33 (1): 25-42.

[16] Bertini, R. L. 2003. "Toward the Systematic Diagnosis of Freway Bottleneck Activation." In Proceedings of IEEE 6th International Conference on Intelligent Transportation Systems, 1-6.
[17] Wieczorek, J., Fernández-Moctezuma, R. J., and Bertini, R. L. 2010. "Techniques for Validating an Automatic Bottleneck Detection Tool Using Archived Freeway Sensor Data." Transportation Research Record: Journal of the Transportation Research Board 2160 (2010): 87-95.

[18] Chen, C., Skabardonis, A., and Varaiya, P. 2003. "Systematic Identification of Freeway Bottlenecks." Transportation Research Record 1867 (1): 46-52.

[19] Juran, J. Prashker, S. Bekhor, I. Ishai, A dynamic traffic assignment model for the assessment, Transportation Research Part C: Emerging Technologies 17 (3) (2009) 240-258.

[20] Acevedo, J., Echeverry, J. C., Bocarejo, J. P., Lleras, G., Ospina, G., and Rodríguez, A. 2009. El Transporte como Soporte al Desarrollo de Colombia: Una Visión al 2040. Bogotá: Ediciones Uniandes. (in Spanish)

[21] Rodriguez-Valencia, A., and Acevedo, J. 2012. Taxi! El modo Olvidado de la Movilidad en Bogotá. Bogotá: Universidad de Los Andes. (in Spanish)

[22] Bocarejo, J., Bravo, G. E., Castro, H. E., Rodríguez, Á., Pérez, P. F., Velásquez, S., and Bautista, L. J. 2011. Prototipo de un Sistema de Información de Tráfico y Movilidad Urbana para la Ciudad de Bogotá, Colombia. Bogotá: Universidad de los Andes, 2011.

[23] G. Bravo C., H. Castro B., L. J. Bautista M., J. P. Bocarejo S., Á. Rodríguez-Valencia, P. F. Pérez-Arteaga, and S. Velázquez G. 2013. "Retos Tecnológicos para Medir el Tráfico en Bogotá Mediante GPS y Comunicación Celuar." Revista de Ingeniería 38: 45-50. (in Spanish) 